\title{
Effects of spin imbalance on the electric-field-driven quantum dissipationless spin current in $p$-doped semiconductors
}

\author{
Liangbin $\mathrm{Hu},{ }^{1,2} \mathrm{Ju} \mathrm{Gao},{ }^{1}$ and Shun-Qing Shen ${ }^{1}$ \\ ${ }^{1}$ Department of Physics, The University of Hong Kong, Pokfulam Road, Hong Kong, People's Republic of China \\ ${ }^{2}$ Department of Physics, South China Normal University, Guangdong 510631, People's Republic of China
}

(Received 22 March 2004; revised manuscript received 30 June 2004; published 17 December 2004)

\begin{abstract}
It was proposed recently by Murakami et al. [Science 301, 1348 (2003)] that in a large class of $p$-doped semiconductors, an applied electric field can drive a quantum dissipationless spin current in the direction perpendicular to the electric field. In this paper we investigate the effects of spin imbalance on this intrinsic spin Hall effect. We show that in a real sample with boundaries, due to the presence of spin imbalance near the edges of the sample, the spin Hall conductivity is not a constant but a sensitively position-dependent quantity, and due to this fact, in order to take the effects of spin imbalance properly into account, a microscopic calculation of both the quantum dissipationless spin Hall current and the spin accumulation on an equal footing is thus required. Based on such a microscopic calculation, a detailed discussion of the effects of spin imbalance on the intrinsic spin Hall effect in thin slabs of $p$-doped semiconductors are presented.
\end{abstract}

DOI: $10.1103 /$ PhysRevB.70.235323

PACS number(s): 73.43.-f, 72.25.Dc, 72.25.Hg, 85.75.-d

\section{INTRODUCTION}

Efficient injection and coherent control of spins in nonmagnetic semiconductors represent two principal challenges in the emerging field of spintronics, a paradigm of semiconductor electronics based on the utilization of the electron's spin degree of freedom. ${ }^{1}$ At a first glance, it seems a trivial thing to inject spins into nonmagnetic semiconductors by use of ferromagnetic metals as sources. However, in reality it is not practical because most of the spin polarizations will be lost at the interface between metal and semiconductor due to the large conductivity mismatch..$^{2,3}$ A possible approach that can solve this problem is to replace ferromagnetic metals by ferromagnetic semiconductors (such as $\mathrm{Ga}_{1-x} \mathrm{Mn}_{x} \mathrm{As}$ ) as sources of spin injection, ${ }^{4-6}$ but for practical use at room temperature, the Curie temperatures of ferromagnetic semiconductors are still too low. Due to such difficulties, how to achieve efficient injection of spins into nonmagnetic semiconductors at room temperature remains an open question and more great efforts are still needed. Recently, based on the Luttinger effective Hamiltonian, ${ }^{7}$ Murakami et al. theoretically predicted that an extraordinary spin Hall effect may occur in a large class of $p$-doped semiconductors (such as $\mathrm{Si}$, $\mathrm{Ge}$, and GaAs), which means that in such a semiconductor, an applied electric field can drive a substantial amount of quantum dissipationless spin current in the direction perpendicular to the electric field, and the spin current does not decrease substantially even at room temperature. ${ }^{8,9}$ This effect might reveal a new way for achieving efficient spin injection in nonmagnetic semiconductors at room temperature without the need of ferromagnetic metals and may also find some other important applications in spintronics. Prior to the discovery of this effect, a similar effect was also predicted by Hirsch $^{10}$ and discussed extensively by several other authors. ${ }^{11,12}$ From the theoretical points of view, the effect conceived by Hirsch is an extrinsic spin Hall effect, which is caused by the spin-orbit dependent anisotropic scatterings from impurities but not an intrinsic property of a material, and it will disappear completely in the absence of impurity scatterings. The spin current generated by the extrinsic spin Hall effect was shown to be rather small, ${ }^{10-12}$ so it is of little use in the problem of spin injections in nonmagnetic semiconductors. Unlike the extrinsic spin Hall effect, the spin Hall effect proposed in Refs. 8 and 9 is purely intrinsic, which arises from the intrinsic spin-orbit coupling in the valence bands of $p$-doped semiconductors and does not rely on any spin-orbit-dependent anisotropic scatterings from impurities. From a more profound point of view, this effect has a deep topological character and shares some basic features with the quantum Hall edge current both physically and mathematically. ${ }^{8,9}$ For example, just like the case of quantum Hall effect, ${ }^{13-15}$ the spin Hall conductivity due to this effect is a dissipationless transport coefficient and can be expressed as an integral over all states below the Fermi energy, and the contribution of each state can be expressed entirely in terms of the curvature of a gauge field in momentum space. ${ }^{8,9}$ Due to such features, the spin current generated by this intrinsic spin Hall effect can be very large (comparable to the ordinary charge currents) and, hence, can serve as an effective source for efficient injections of spins in nonmagnetic semiconductors at room temperature. Very recently, a similar intrinsic spin Hall effect was also found by Sinova et al. in two-dimensional electron gases (2DEGs) with Rashba spinorbit coupling. ${ }^{16}$ They found that in 2DEGs with Rashba spin-orbit coupling, the dissipationless and intrinsic spin Hall conductivity will take a universal value as long as both spinorbit split bands are occupied. It is anticipated this effect will also find some important applications in the emerging field of spintronics.

Although some basic concepts about the intrinsic spin Hall effect are clear, ${ }^{8,9,16}$ there are still a number of important questions which are needed to be further clarified, and in the last year many theoretical works have been devoted to the study of this extraordinary effect. ${ }^{17-27}$ Basically, most of these theoretical works have been focused on the calculation of the intrinsic spin Hall conductivity. In the present paper, 
we present a theoretical investigation on the effects of spin imbalance on the intrinsic spin Hall effect in $p$-doped semiconductors. While it was well known both experimentally and theoretically that in spin-polarized transport phenomena ${ }^{28-32}$ (including in semiconductor spintronics devices $)^{33-35}$ spin imbalance may have significant influences on the transports of spins, what influences spin imbalance will have on the intrinsic spin Hall effect is still a new subject and has not yet been explored. For the intrinsic spin Hall effect, from both the experimental and theoretical points of view, a clear understanding of the effects of spin imbalance would be much desirable because spin imbalance may not only have some significant influences on the electric-field driven quantum dissipationless spin current and on its practical applications but also play a crucial role in the experimental measurement of the effect. ${ }^{8}$ In this paper, based on a solid microscopic ground, we will derive a set of selfconsistent spin transport equations which will present a proper description on the interplay between the spin imbalance and the electric-field driven quantum dissipationless spin current in the intrinsic spin Hall effect in $p$-doped semiconductors. Starting from these spin transport equations and with the help of appropriate boundary conditions, the quantum dissipationless spin current and the induced nonequilibrium spin accumulation in an actual sample with boundaries can be calculated simultaneously on an equal footing. Our results show that the characteristics of the interplay between the quantum dissipationless spin current and the spin imbalance in the intrinsic spin Hall effect in $p$-doped semiconductors are very different from what was found in usual spinpolarized transport phenomena (including in the extrinsic spin Hall effect), and some usual concepts about the interplay between spin current and spin imbalance cannot be applied to the intrinsic spin Hall effect.

The paper is organized as follows: In Sec. II, we will present a microscopic derivation of the spin transport equations for describing the intrinsic spin Hall effect in $p$-doped semiconductors. In our derivation, the effects of spin imbalance will be included explicitly. In Sec. III by solving these spin transport equations with the help of appropriate boundary conditions, the electric-field driven quantum dissipationless spin current and the induced nonequilibrium spin accumulation in thin slabs of $p$-doped semiconductors will be calculated explicitly.

\section{SPIN TRANSPORT EQUATIONS IN THE PRESENCE OF SPIN IMBALANCE}

In a large class of $p$-doped semiconductors such as $\mathrm{Si}, \mathrm{Ge}$, and GaAs, the valence bands are fourfold degenerate at the $\Gamma$ point. In the momentum representation and taking the hole picture, the valance bands in such semiconductors can be described by the following Luttinger effective Hamiltonian: $7^{7,8}$

$$
\hat{H}_{0}=\frac{\hbar^{2}}{2 m}\left[\left(\gamma_{1}+\frac{5}{2} \gamma_{2}\right) \mathbf{k}^{2}-2 \gamma_{2}(\mathbf{k} \cdot \mathbf{S})^{2}\right],
$$

where $S_{i}$ is the spin-3/2 matrix, $\gamma_{1}$ and $\gamma_{2}$ are the Luttinger parameters. For a given wave vector $\mathbf{k}$, the Hamiltonian (1) has two eigenvalues, given by

$$
\begin{aligned}
& \epsilon_{H}(\mathbf{k})=\epsilon_{\lambda= \pm 3 / 2}(\mathbf{k})=\frac{\gamma_{1}-2 \gamma_{2}}{2 m} \hbar^{2} k^{2} \equiv \frac{\hbar^{2} k^{2}}{2 m_{H}}, \\
& \epsilon_{L}(\mathbf{k})=\epsilon_{\lambda= \pm 1 / 2}(\mathbf{k})=\frac{\gamma_{1}+2 \gamma_{2}}{2 m} \hbar^{2} k^{2} \equiv \frac{\hbar^{2} k^{2}}{2 m_{L}},
\end{aligned}
$$

where $\lambda \equiv \hbar^{-1} \mathbf{k} \cdot \mathbf{S} / k$ is a good quantum number of the Hamiltonian $\hat{H}_{0}$. The hole bands described by Eqs. (2) and (3) are referred to as the light-hole (LH) and heavy-hole (HH) bands, respectively. When a uniform electric field $\mathbf{E}$ is applied, the full Hamiltonian will be given by $\hat{H}=\hat{H}_{0}$ $+e \mathbf{E} \cdot \mathbf{x}$, where $-e$ is the charge of an electron. The equation of motion for the light and heavy holes in a uniform electric field has been derived in much detail in Ref. 8, and in the semiclassical approximation (i.e., the spin is treated as a classical variable and hence commutes with the current operator), the following equation of motion was obtained therein:

$$
\dot{k}_{i}=\frac{e E_{i}}{\hbar}, \quad \dot{x}_{i}=\frac{\hbar k_{i}}{m_{\lambda}}+\epsilon_{i j l} \lambda\left(2 \lambda^{2}-\frac{7}{2}\right) \frac{k_{l}}{k^{3}} \dot{k}_{j},
$$

where $\epsilon_{i j l}$ is the usual fully antisymmetric tensor in three dimensions. The occurrence of the last term in Eq. (4) is unusual, it represents a "Lorentz force" in momentum space and is a natural generalization of the quantum Hall effect ${ }^{13-15}$ to three dimensions. ${ }^{8,9}$ It is just due to this "Lorentz force" in momentum space (which makes the hole velocity noncollinear with its momentum) that the applied electric field will drive a quantum dissipationless spin Hall current in the direction perpendicular to the electric field. From Eq. (4), one can get that in the low temperature limit and in the semiclassical approximation, the net spin current due to both the LH and $\mathrm{HH}$ bands will be given by ${ }^{8}$

$$
J_{j}^{i}=\frac{\hbar}{3} \sum_{\lambda, \mathbf{k}} \dot{x}_{j} \frac{\lambda k_{i}}{k} n_{\lambda}(\mathbf{k})=\sigma_{s}^{0} \epsilon_{i j k} E_{k},
$$

where $J_{j}^{i}$ denotes the net spin current following to the $x_{j}$ direction with spin parallel to the $x_{i}$ direction, $n_{\lambda}(\mathbf{k})$ is the filling of holes in the band with helicity $\lambda$, and $\sigma_{s}^{0}$ is the spin Hall conductivity, which is given by

$$
\sigma_{s}^{0}=\frac{e}{12 \pi^{2}}\left(3 k_{H}^{F}-k_{L}^{F}\right),
$$

with $k_{L}^{F}$ and $k_{H}^{F}$ denoting the Fermi wave numbers in the LH and $\mathrm{HH}$ bands, respectively. In obtaining Eqs. (5) and (6), one has assumed that the fillings of holes in each band can be described by the simple Fermi-Dirac equilibrium distribution function. An alternative way of calculating the intrinsic spin Hall conductivity is by use of the Kubo formula. ${ }^{9,16-18,21,22}$ Based on the Kubo formula, as was shown in Ref. 9, the full quantum treatment of the noncommutativity between the quantum spin and current operator will lead to a quantum correction to Eq. (6). But if one takes the semiclassical limit, the result will become the same as was given by Eq. (6).

Equations (5) and (6) are the central results of Refs. 8 and 9. They are valid in the absence of spin imbalance. But in a real sample with boundaries, when a spin current circulates in it, spin imbalance will be caused inevitably near the edge 
of the sample by the spin current, and in the presence of spin imbalance, the spin current may be significantly different from what was given by Eqs. (5) and (6), especially in the regions near the edges of the sample. The reason for this is that in the presence of spin imbalance, the fillings of holes in the $\mathrm{LH}$ and $\mathrm{HH}$ bands may deviate significantly from the corresponding cases in the equilibrium state. In order to take the effects of spin imbalance properly into account, one should obtain the distribution function strictly by solving the Boltzman transport equation, which describes the changes of the distribution function in a nonequilibrium state. In a nonequilibrium but steady state, the Boltzman equation reads

$$
\dot{\mathbf{x}} \cdot \nabla f_{\lambda}(\mathbf{x}, \mathbf{k})+\dot{\mathbf{k}} \cdot \nabla_{\mathbf{k}} f_{\lambda}(\mathbf{x}, \mathbf{k})=-\sum_{\lambda^{\prime}}\left(\frac{\partial f_{\lambda}}{\partial t}\right)_{\lambda \rightarrow \lambda^{\prime}}^{\text {(coll. })},
$$

where $\left(\partial f_{\lambda} / \partial t\right)_{\lambda \rightarrow \lambda^{\prime}}^{\text {(coll.) }}$ is the collision term due to impurity scatterings, and $\dot{\mathbf{x}}$ and $\dot{\mathbf{k}}$ are the drift velocities of holes in the real space and in the momentum space, respectively. Similar to Ref. 8, in this paper we will confine our discussion to the semiclassical limit and weak external electric field (i.e., in the linear response regime) so that the semiclassical equation of motion given by Eq. (4) can be applied. ${ }^{8,9}$ The collision term $\left(\partial f_{\lambda} / \partial t\right)_{\lambda \rightarrow \lambda^{\prime}}^{\text {(coll.) }}$ will be given by

$$
\begin{aligned}
\left(\frac{\partial f_{\lambda}}{\partial t}\right)_{\lambda \rightarrow \lambda^{\prime}}^{(\text {coll. })}= & -\int \frac{d^{3} \mathbf{k}^{\prime}}{(2 \pi)^{3}} w_{\lambda, \lambda^{\prime}}^{(i)}\left(\mathbf{k}, \mathbf{k}^{\prime}\right) \delta\left[\epsilon_{\lambda}(\mathbf{k})-\epsilon_{\lambda^{\prime}}\left(\mathbf{k}^{\prime}\right)\right] \\
& \times\left[f_{\lambda}(\mathbf{x}, \mathbf{k})-f_{\lambda^{\prime}}\left(\mathbf{x}, \mathbf{k}^{\prime}\right)\right],
\end{aligned}
$$

where $w_{\lambda, \lambda^{\prime}}^{(i)},\left(\mathbf{k}, \mathbf{k}^{\prime}\right)$ is the probability of a hole to be scattered from the state $|\mathbf{k} \lambda\rangle$ into the state $\left|\mathbf{k}^{\prime} \lambda^{\prime}\right\rangle$ due to impurity scatterings, and the impurity scatterings will be assumed to be isotropic and spin independent.

In the equilibrium state, the fillings of holes in each band are stable and can be described by the simple Fermi-Dirac equilibrium distribution function. When the external electric field is applied and the system turns into an nonequilibrium but steady state, the fillings of holes in each band will still be stable but different from what was described by the simple Fermi-Dirac equilibrium distribution function. In the presence of spin imbalance, the changes of the fillings of holes in the $\mathrm{LH}$ and $\mathrm{HH}$ bands will be caused primarily by two kinds of contributions. The first kind of contribution is caused by the drifts of holes in the external electric field, and the second kind of contribution is due to the occurrence of spin imbalance. In the linear response regime (i.e., in a weak electric field), the deviations of the fillings of holes in each band from the corresponding cases in the equilibrium state are small, and the two kinds of contributions will be independent and both be proportional to $\partial f^{0} / \partial \epsilon_{F}$ (here $f^{0}=\left(\exp \left\{\beta\left[\epsilon_{\lambda}(\mathbf{k})\right.\right.\right.$ $\left.\left.\left.-\epsilon_{F}\right]\right\}+1\right)^{-1}$ is the usual Fermi-Dirac equilibrium distribution function with $\beta$ denoting the inverse of temperature and $\epsilon_{F}$ the Fermi level in the equilibrium state). Considering this fact and by use of the relaxation time approximation, in the linear response regime the nonequilibrium distribution function $f_{\lambda}(\mathbf{x}, \mathbf{k})$ (in a nonequilibrium but steady state) can be expressed as the following:

$$
f_{\lambda}(\mathbf{x}, \mathbf{k})=f^{0}+\mu_{\lambda}(\mathbf{x}) \frac{\partial f^{0}}{\partial \epsilon_{F}}+e \tau\left[\mathbf{E}_{\lambda}(\mathbf{x}) \cdot \mathbf{V}_{\lambda}\right] \frac{\partial f^{0}}{\partial \epsilon_{F}},
$$

where $\mathbf{V}_{\lambda}=\hbar \mathbf{k} / m_{\lambda}$ is the velocity of holes, $\tau$ is the total relaxation time of holes due to impurity-induced random scatterings, and $\mathbf{E}_{\lambda}(\mathbf{x})$ is the total effective field felt by a moving hole in the band with helicity $\lambda$, which is the sum of the external electric field $\mathbf{E}$ and a band-dependent effective field induced by the spin imbalance in the sample. The detailed definition of $\mathbf{E}_{\lambda}(\mathbf{x})$ and $\tau$ will be given later. The second term in Eq. (9) just characterizes the deviation of the filling of holes in the band with helicity $\lambda$ from the corresponding case in the equilibrium state due to the occurrence of spin imbalance in the sample, and the presence of this term is mathematically equivalent to introducing a band-dependent "shift" $\mu_{\lambda}$ in the Fermi level $\epsilon_{F}$. (It should be noted that unlike the corresponding cases in usual spin-polarized transport phenomena, here $\mu_{\lambda}$ does not relate directly to the spin accumulation because the label $\lambda$ does not correspond to a fixed spin-polarization direction in real space.) The third term in Eq. (9) denotes the change of the filling due to the drifts of holes in the external electric field and in the presence of impurity scatterings. By inserting Eq. (9) into Eq. (7) and assuming that the impurity scatterings are isotropic, the Boltzman equation can be simplified to the following form:

$$
\begin{aligned}
\mathbf{V}_{\lambda} \cdot\left[\mathbf{E}+\frac{1}{e} \nabla \mu_{\lambda}(\mathbf{x})+\tau \nabla\left[\mathbf{E}_{\lambda}(\mathbf{x}) \cdot \mathbf{V}_{\lambda}\right]\right] \\
=\sum_{\lambda^{\prime}(\neq \lambda)} \frac{\mu_{\lambda}(\mathbf{x})-\mu_{\lambda^{\prime}}(\mathbf{x})}{e \tau_{\lambda \lambda^{\prime}}}+\sum_{\lambda^{\prime}} \frac{\tau \mathbf{E}_{\lambda}(\mathbf{x}) \cdot \mathbf{V}_{\lambda}}{\tau_{\lambda \lambda^{\prime}}},
\end{aligned}
$$

where $\tau_{\lambda \lambda^{\prime}}$ is a characteristic relaxation time defined by

$$
\tau_{\lambda \lambda^{\prime}}=\left[\int \frac{d^{3} \mathbf{k}^{\prime}}{(2 \pi)^{3}} w_{\lambda, \lambda^{\prime}}^{(i)}\left(\mathbf{k}, \mathbf{k}^{\prime}\right) \delta\left[\epsilon_{\lambda}(\mathbf{k})-\epsilon_{\lambda^{\prime}}\left(\mathbf{k}^{\prime}\right)\right]\right]^{-1},
$$

which characterizes the probability (given by $\tau_{\lambda \lambda^{\prime}}^{-1}$ ) for a hole in the band with helicity $\lambda$ to be scattered into the band with helicity $\lambda^{\prime}$ due to impurity scatterings. For simplicity, in the following we will assume that the intraband-scattering relaxation time $\tau_{\lambda \lambda} \equiv \tau_{1}$ (independent of $\lambda$ ) and the interbandscattering relaxation time $\tau_{\lambda \lambda^{\prime}(\neq \lambda)} \equiv \tau_{2}$ (independent of $\lambda$ and $\lambda^{\prime}$ ), and as usual, the total scattering probability for a hole (given by $\tau^{-1}$, i.e., the inverse of the total relaxation time of a hole) can be given by

$$
\tau^{-1}=\sum_{\lambda^{\prime}} \tau_{\lambda \lambda^{\prime}}^{-1}=\frac{1}{\tau_{1}}+\frac{3}{\tau_{2}}
$$

which is assumed to be independent of the band label $\lambda$. Multiplying both sides of Eq. (10) by $\mathbf{V}_{\lambda}$ and then integrating both sides with respect to $\mathbf{V}_{\lambda}$ and with the help of Eq. (12), one can find that the total effective field felt by a moving hole in the band with helicity $\lambda$ should be given by 


$$
\mathbf{E}_{\lambda}(\mathbf{x})=\mathbf{E}+\frac{1}{e} \nabla \mu_{\lambda}(\mathbf{x}) .
$$

Equation (13) suggests that in the presence of spin imbalance, in addition to the external electric field $\mathbf{E}$, conduction electrons will also feel an effective field proportional to the gradient of the band- and position-dependent shift in the Fermi level. After $\tau$ and $\mathbf{E}_{\lambda}(\mathbf{x})$ are determined from Eqs. (12) and (13), the nonequilibrium distribution function $f_{\lambda}(\mathbf{x}, \mathbf{k})$ will also be determined by Eq. (9). Then in the semiclassical limit the electric-field driven quantum dissipationless spin current can be obtained through the following formula:

$$
J_{j}^{i}(\mathbf{x})=\sum_{\lambda} \int \frac{d^{3} \mathbf{k}}{(2 \pi)^{3}}\left[\dot{x}_{j} s_{i, \lambda}(\mathbf{k})\right] f_{\lambda}(\mathbf{x}, \mathbf{k}),
$$

where $\dot{x}_{j}=\hbar k_{j} / m_{\lambda}+\epsilon_{j k l} \lambda\left(2 \lambda^{2}-7 / 2\right) k_{l} \dot{k}_{k} / k^{3}$ [see Eq. (4)] and $s_{i, \lambda}(\mathbf{k})=\hbar\left(\lambda k_{i} / k\right) / 3$ are the velocity and the spin of a hole with momentum $\mathbf{k}$ and helicity $\lambda$, respectively. [Since the spin-3/2 matrix $\mathbf{S}$ in the Hamiltonian (1) is a summation of the spin angular momentum $\mathbf{s}$ with spin one-half and the atomic orbital angular momentum $\mathbf{I}$ with spin one, the expectation value of $\mathbf{s}$ should be one-third of $\mathbf{S} .^{7,8}$ By substituting Eqs. (12) and (13) into Eq. (9) and then inserting Eq. (9) into Eq. (14), the following result can be obtained:

$$
J_{j}^{i}(\mathbf{x})=\sigma_{s}(\mathbf{x}) \epsilon_{i j k} E_{k},
$$

where $\sigma_{s}(\mathbf{x})$ is the spin Hall conductivity in the presence of spin imbalance, which is given by

$$
\sigma_{s}(\mathbf{x})=\sigma_{s}^{0}-\frac{e}{48 \pi^{2} \epsilon_{F}}\left[3 k_{H}^{F} \mu_{H}(\mathbf{x})-k_{L}^{F} \mu_{L}(\mathbf{x})\right] .
$$

Here $\sigma_{s}^{0}$ is the spin Hall conductivity in the absence of spin imbalance, which has been defined in Eq. (6), and $\mu_{H}(\mathbf{x})$ $\equiv \mu_{3 / 2}(\mathbf{x})+\mu_{-3 / 2}(\mathbf{x})$ and $\mu_{L}(\mathbf{x}) \equiv \mu_{1 / 2}(\mathbf{x})+\mu_{-1 / 2}(\mathbf{x})$. Equations (15) and (16) show that the effects of spin imbalance on the quantum dissipationless spin current due to the intrinsic spin Hall effect in $p$-doped semiconductors are very different from what was found in usual spin-polarized phenomena (including the extrinsic spin Hall effect). ${ }^{10-12}$ First, in the presence of spin imbalance, the spin Hall conductivity due to the intrinsic spin Hall effect in $p$-doped semiconductors might not be a constant but a position-dependent quantity. This is a feature that was not seen before. Second, for the intrinsic spin Hall effect in $p$-doped semiconductors, the change of the quantum dissipationless spin current due to the occurrence of spin imbalance is determined directly by $\mu_{\lambda}(\mathbf{x})$ (i.e., the band-dependent "shifts" in the Fermi level) but is independent of the gradients of $\mu_{\lambda}(\mathbf{x})$. This is also significantly different from what was found in usual spin-polarized transports (including the extrinsic spin Hall effect). These unusual characteristics of the intrinsic spin Hall effect in $p$-doped semiconductors can be understood by the following arguments. According to Eq. (6), the spin Hall conductivity should be determined uniquely by the Fermi wave numbers $k_{H}^{F}$ and $k_{L}^{F}$. In the presence of spin imbalance, because the spin imbalance will induce a position- and band-dependent shift $\mu_{\lambda}(\mathbf{x})$ in the Fermi level, the Fermi wave numbers $k_{H}^{F}$ and $k_{L}^{F}$ will also be position dependent, and the changes of $k_{H}^{F}$ and $k_{L}^{F}$ due to the occurrence of spin imbalance will be determined directly by $\mu_{\lambda}(\mathbf{x})$. Due to this reason, in the presence of spin imbalance, the spin Hall conductivity will be a position-dependent quantity, and the change of the spin Hall current due to the occurrence of spin imbalance will be determined by $\mu_{\lambda}(\mathbf{x})$ but independent of the gradients of $\mu_{\lambda}(\mathbf{x})$. Finally, it should be pointed out that because we have considered only isotropic and spinless impurity scattering, the mechanism of the generation of the spin Hall current described by Eqs. (15) and (16) is still purely intrinsic, though there are some significant differences between Eq. (16) and Eq. (6). In fact, one can check that in the linear response regime the impurity scattering term (i.e., the third term) in Eq. (9) does not contribute to the spin Hall conductivity given by Eq. (16). This point will be more clearly seen from the results presented in Sec. III. Of course, if the impurity scatterings are spin-orbit dependent, then the total spin current will contain not only the intrinsic part but also contain an extrinsic part due to the spin-orbit-dependent impurity scatterings through the mechanism proposed by Hirsch. ${ }^{10-12}$

In the ordinary charge Hall effect, the charge Hall current causes charge imbalance in a sample and results in charge accumulation. Similarly, in the spin Hall effect, the spin imbalance caused by the spin Hall current will result in nonequilibrium spin accumulation in a sample. Corresponding to the quantum spin Hall current given by Eqs. (15) and (16), the nonequilibrium spin accumulation induced by the quantum spin Hall current can be obtained as the following:

$$
\begin{aligned}
S_{i}(\mathbf{x}) & =\sum_{\lambda} \int \frac{d^{3} \mathbf{k}}{(2 \pi)^{3}} s_{i, \lambda}(\mathbf{k}) f_{\lambda}(\mathbf{x}, \mathbf{k}) \\
& =\epsilon_{i j l} \frac{E_{l} \hbar^{2}}{16 e \epsilon_{F}^{2}} \frac{\partial}{\partial x_{j}}\left[C_{L} \mu_{L}(\mathbf{x})-3 C_{H} \mu_{H}(\mathbf{x})\right],
\end{aligned}
$$

where $C_{L}=e^{2} \tau\left(k_{L}^{F}\right)^{3} / 6 \pi^{2} m_{L}$ and $C_{H}=e^{2} \tau\left(k_{H}^{F}\right)^{3} / 6 \pi^{2} m_{H}$ are the ordinary charge conductivities of the light holes and the heavy holes, respectively. Equations (15)-(17) show that in the intrinsic spin Hall effect in $p$-doped semiconductors, both the quantum dissipationless spin current and the spin accumulation will depend sensitively on $\mu_{\lambda}(\mathbf{x})$, i.e., the banddependent shifts in the Fermi level. To find out the equations that $\mu_{\lambda}(\mathbf{x})$ should satisfy, one can substitute Eqs. (12) and (13) into Eq. (10) and integrate both sides of Eq. (10) with respect to $\mathbf{k}$, then one will arrive at the following equation:

$$
\nabla^{2} \mu_{\lambda}(\mathbf{x})=\frac{1}{D_{\lambda}^{2}}\left[4 \mu_{\lambda}(\mathbf{x})-\mu_{H}(\mathbf{x})-\mu_{L}(\mathbf{x})\right],
$$

where $D_{\lambda}=V_{\lambda}^{F} \sqrt{\tau_{2} \tau / 3}$ is a characteristic hole diffusion length and $V_{\lambda}^{F}$ is the band-dependent Fermi velocity. In addition to Eq. (18), $\mu_{\lambda}(\mathbf{x})$ should also satisfy the charge neutrality condition, which requires that the net changes of the charge density due to the flow of the quantum spin current, given by $\delta \rho_{c}=-e \Sigma_{\lambda} \int\left[\left(d^{3} \mathbf{k}\right) /(2 \pi)^{3}\right]\left\{f_{\lambda}(\mathbf{x}, \mathbf{k})-f^{0}\left[\epsilon_{\lambda}(\mathbf{k})\right]\right\}$, should be zero. This leads to the following equation: 


$$
\mu_{H}(\mathbf{x})=-\left(\frac{m_{L}}{m_{H}}\right)^{3 / 2} \mu_{L}(\mathbf{x}) .
$$

Equations (15)-(19) are the central results of the present paper. They constitute a set of self-consistent equations from which both the quantum dissipationless spin current and the spin accumulation due to the intrinsic spin Hall effect in a real sample of $p$-doped semiconductors with boundaries can be obtained simultaneously with the help of appropriate boundary conditions.

\section{INTRINSIC SPIN HALL EFFECT IN THIN SLABS OF $p$-DOPED SEMICONDUCTORS}

Equations (15)-(19) are rather general and in principle they can be applied to samples with any kind of geometries. In the experimental measurement of the Hall effect (including the spin Hall effect), a thin slab geometry (i.e., the Hall bar) is usually applied. In this section, starting from Eqs. (15)-(19), we will present a detailed theoretical investigation on the intrinsic spin Hall effect in a thin slab of $p$-doped semiconductors. We assume that the longitudinal direction of the slab is along the $z$ axis and the transverse direction along the $y$ axis and the normal of the surface along the $x$ axis, respectively, and an external electric field $E_{z}$ is applied in the longitudinal direction of the slab. The thickness of the slab is assumed to be much smaller than the hole diffusion length $D_{\lambda}$ and the length of the slab is assumed to be much larger than the width, so that only spin current flowing to the $y$ direction (i.e., in the transverse direction of the slab) with spin parallel to the $x$ direction need to be considered. The two boundaries of the slab are assumed to be located at $y$ $= \pm w / 2$, and $w$ is the width of the slab. In general, it is very difficult to solve Eqs. (15)-(19) analytically. In order to get some explicit expressions for the spin Hall current and the spin accumulation, we assume that in Eqs. (15)-(19) the hole diffusion length $D_{\lambda}$ is $\lambda$ independent (i.e., $D_{\lambda} \equiv D$ ) and $m_{L}$ $\simeq m_{H}$. Then from Eqs. (18) and (19) one can see that $\mu_{H}(y) \equiv \mu_{3 / 2}(y)+\mu_{-3 / 2}(y)$ and $\mu_{L}(y) \equiv \mu_{1 / 2}(y)+\mu_{-1 / 2}(y)$ can be expressed as

$$
\mu_{H}(y) \simeq-\mu_{L}(y)=A e^{2 y / D}+B e^{-2 y / D},
$$

where $A$ and $B$ are two constant coefficients that need to be determined by the appropriate boundary condition. In this paper, we will consider the transverse open circuit boundary condition. In the transverse open circuit boundary condition, the spin Hall current will be zero at the two boundaries of the slab, i.e., $J_{y}^{x}(y= \pm w / 2)=0$. Substituting Eq. (20) into Eqs. (15) and (16), the spin Hall current $J_{y}^{x}(y)$ can be expressed as a function of the coefficients $A$ and $B$. Then by use of the transverse open circuit boundary condition, the coefficients $A$ and $B$ can be determined, and one can get that

$$
A=B=\frac{2 \epsilon_{F}\left(3 k_{H}^{F}-k_{L}^{F}\right)}{3 k_{H}^{F}+k_{L}^{F}} \frac{1}{\cosh (2 w / D)} .
$$

After the coefficients $A$ and $B$ are determined, the spin Hall current $J_{y}^{x}(y)$ and the spin Hall conductivity $\sigma_{s}(y)$ will also be obtained by inserting Eq. (20) into Eqs. (15) and (16), and the results are given by

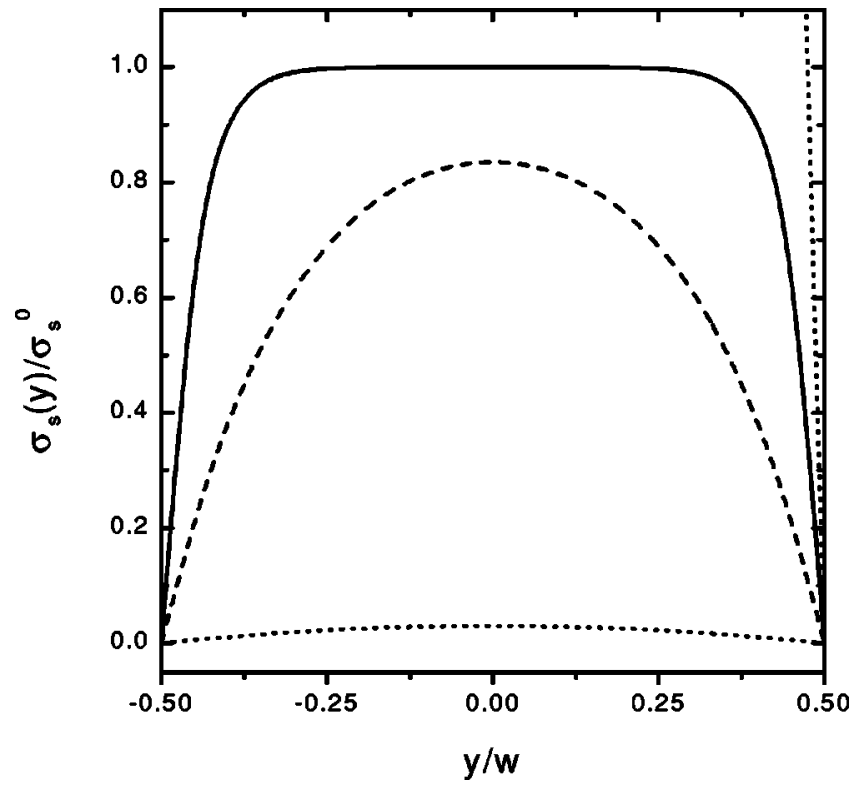

FIG. 1. Illustration of the position dependences of the spin Hall conductivity $\sigma_{s}(y)$ in three cases with different ratios of $w / D$. $[w / D=50$ for the solid line, $w / D=10$ for the dashed line, and $w / D=1$ for the dotted line. $\sigma_{s}(y)$ is normalized by $\sigma_{s}^{0}$, i.e., the spin Hall conductivity in the absence of spin imbalance.]

$$
\begin{gathered}
J_{y}^{x}(y)=\sigma_{s}(y) E_{z} \\
\sigma_{s}(y)=\sigma_{s}^{0}\left[1-\frac{\cosh (2 y / D)}{\cosh (w / D)}\right] .
\end{gathered}
$$

Equations (22) and (23) show that, in the presence of spin imbalance, both the spin Hall current and the spin Hall conductivity might be highly position dependent and might also depend sensitively on the hole diffusion length $D$ and the width $w$ of the sample. The spin Hall conductivity $\sigma_{s}(y)$ will be maximum at the center of the sample (i.e., at $y=0$ ) and tend to be zero at the edges of the sample. Two limiting cases will be especially interesting. The first case is that the hole diffusion length $D$ is much larger than the width $w$ of the sample. In this limiting case the spin Hall current will be very small, i.e., $\sigma_{s}(y) \simeq 0$ everywhere. The second interesting case is that $w \gg D$. In this limiting case, the maximum value of the spin Hall conductivity will be given by $\sigma_{s}(y) \simeq \sigma_{s}^{0}$ (at $y=0)$ and $\sigma_{s}(y) \rightarrow 0$ as $y \rightarrow \pm w / 2$. These features can be seen clearly from Fig. 1, where we have plotted the position dependence of the spin Hall conductivity $\sigma_{s}(y)$ in three cases with different ratios of $w / D$. From Fig. 1 and Eq. (23), one can see clearly that if no boundaries exist (i.e., $w \rightarrow \infty$ and, hence, no spin imbalance occurs), the spin Hall conductivity will be a constant and return to the same result as was given by Eq. (6), i.e., the spin Hall conductivity will not be changed by weak isotropic and spinless impurity scatterings. This is in agreement with Ref. 8 and also in agreement with the result obtained by a more accurate calculation performed in Ref. 20. It is interesting to note that recently a similar conclusion was also obtained for the intrinsic spin Hall effect in 2DEGs with Rashba spin-orbit coupling by both numeri- 
cal simulations ${ }^{24}$ and analytical calculations, ${ }^{26,27}$ which suggest that in the presence of weak (isotropic and spinless) impurity scatterings, the intrinsic spin Hall conductivity in a Rashba two-dimensional electron gas should still take a universal value, proving that the sample size exceeds the localization length..$^{24,26,27}$ Of course, it should be pointed out that at present different views also exist on this problem. For example, in Ref. 25 it was argued that the spin-orbitcoupling induced intrinsic spin Hall current in a Rashba twodimensional electron gas should vanish in the presence of impurity scatterings, even if the impurity scatterings are weak and spinless.

The quantum dissipationless spin current generated by the intrinsic spin Hall effect does not carry charges (i.e., it is a pure spin current), so it is very difficult to measure the quantum dissipationless spin current directly. An indirect but much more convenient way to detect the quantum dissipationless spin current is to measure the nonequilibrium spin accumulation induced by the quantum dissipationless spin current. The nonequilibrium spin accumulation induced by the quantum dissipationless spin Hall current in a thin slab of $p$-doped semiconductors can be got by inserting Eqs. (20) and (21) into Eq. (17), and the following result can be obtained:

$$
S_{x}(y)=\frac{3 \pi^{2} \hbar^{2} \sigma_{s}^{0} E_{z}\left(C_{L}+3 C_{H}\right) \sinh (2 y / D)}{e^{2} \epsilon_{F}\left(k_{L}^{F}+3 k_{H}^{F}\right) D \cosh (w / D)} .
$$

Equation (24) shows that the spin accumulation will be linearly proportional to the spin Hall conductivity $\sigma_{s}^{0}$ and also depend sensitively on the ordinary charge conductivities $C_{L}$ and $C_{H}$ of the light and heavy holes. It also have a sensitive dependence on the hole diffusion length $D$ and the sample width $w$. According to Eq. (24), for a infinitely large sample without boundaries (i.e., $w \rightarrow \infty$ ), no spin accumulation will appear [i.e., $S_{x}(y)=0$ for any finite $y$ ]. This is different from what was found in a Rashba two-dimensional electron gas, where it was found that the application of an in-plane electric field would induce a homogeneous nonequilibrium spin accumulation without resort to the boundary effects. ${ }^{36-38}$ From Eq. (24), one can see that for an actual sample with boundaries, the spatial distribution of the spin accumulation due to the intrinsic spin Hall effect would be highly inhomogeneous. The spin accumulation will be maximum at the edges of the sample and vanish near the center of the slab, and the spin accumulation at the edges of the sample will increase with the increase of the sample width $w$. This has been illustrated in Fig. 2. From Fig. 2 one can see that if the sample width $w$ is much smaller than the hole diffusion length $D$, the spin accumulation induced by the quantum spin Hall current will be very small. On the other hand, if the sample width $w$ is much larger than the hole diffusion length $D$, the spin accumulation at the edges of the sample will be almost a constant, independent of the sample width. This will be a merit for the experimental measurement of the intrinsic spin Hall effect. To obtain a quantitative estimation on the order of the magnitude of the spin accumulation induced by the quantum dissipationless spin Hall current in a real sample, let us consider some actual experimental parameters. We take

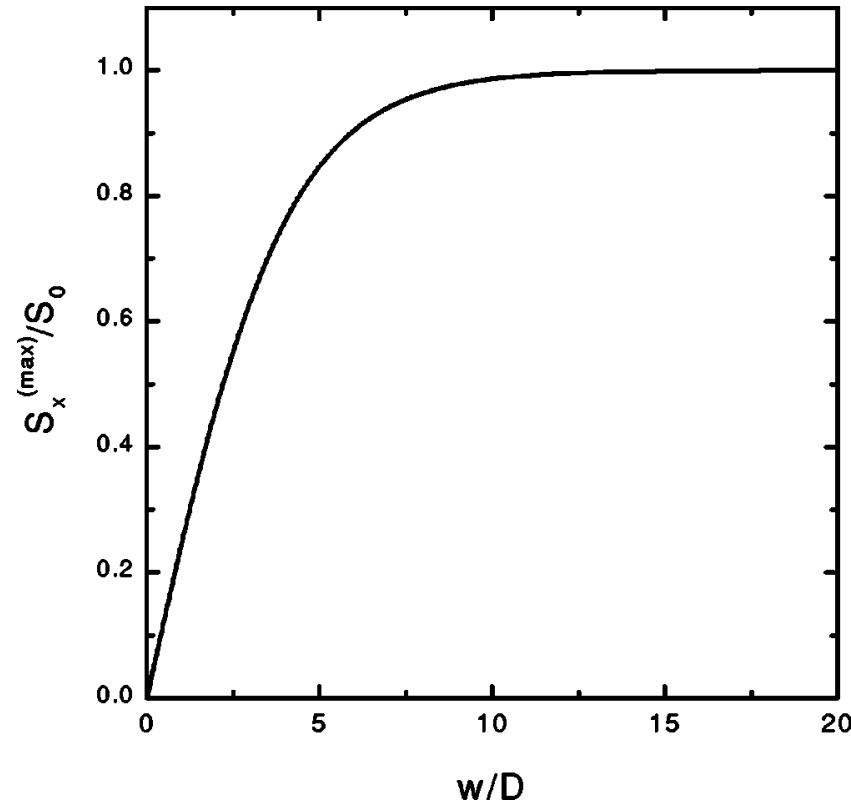

FIG. 2. Illustration of the changes of the spin accumulation at the edges of a sample with the variation of the sample width. \{The spin accumulation is normalized by $S_{0}=\left[3 \pi^{2} \hbar^{2} \sigma_{s}^{0} E_{z}\left(C_{L}\right.\right.$ $\left.\left.\left.+3 C_{H}\right) / e^{2} \epsilon_{F} D\left(k_{L}^{F}+3 k_{H}^{F}\right)\right]\right\}$.

the ordinary conductivity $C_{L, H} \sim 10^{2} \Omega^{-1} \mathrm{~cm}^{-1}$ and the hole diffusion length $D \sim 10 \mathrm{~nm}$ and $\hbar / \epsilon_{F} \sim 1$ fs. These parameters are typical of the holes in GaAs with the hole density $n \sim 10^{19} \mathrm{~cm}^{-3}$. The width $w$ of the sample is assumed to be $100 \mathrm{~nm}$ (much larger than the hole diffusion length) and a current density $j_{x} \sim 10^{4} \mathrm{~A} / \mathrm{cm}^{2}$. By use of the parameters listed earlier, from Eq. (24) it can be estimated that the spin accumulation at the edges of the sample will be on the order of $10^{13}-10^{15} \mu_{B} \mathrm{~cm}^{-2}$. Such magnitudes should be large enough to be measured by some ordinary experimental methods, for example, by the method proposed in Refs. 8-11. Finally, it should be pointed out that a rough estimation of the spin accumulation due to the quantum dissipationless spin Hall current was also presented in the supporting online material for Ref. 8 based on a simple analysis by use of the usual spin diffusion equation, but there are some significant differences between the results obtained in the present paper and the corresponding results reported therein. This can be seen by making a comparison between Eq. (24) obtained in the present paper and Eq. (S16) presented in the supporting online material for Ref. 8. For example, according to Eq. (24) obtained in the present paper, the spin accumulation will not only depend on the spin Hall conductivity but also depend sensitively on the ordinary charge conductivities of the light and heavy holes; however, according to Eq. (S16) in the supporting online material for Ref. 8, the spin accumulation will only depend on the spin Hall conductivity but is independent of the ordinary charge conductivities of the light and heavy holes. Our results show that though the mechanism of the intrinsic spin Hall effect is purely intrinsic, impurity scatterings might have some significant influences on the effect in a real sample with boundaries, and the usual spin diffusion equation might not be very suitable for describing this effect. In fact, from the microscopic calculation presented in Sec. II, 
one can see that in general the quantum dissipationless spin Hall current [given by Eqs. (15) and (16)] and the spin accumulation [given by Eq. (17)] due to the intrinsic spin Hall effect do not satisfy the usual spin diffusion equation.

In conclusion, in this paper we have presented a detailed theoretical investigation on the effects of spin imbalance on the intrinsic spin Hall effect in $p$-doped semiconductors. We have shown that in a real sample with boundaries, the spin Hall conductivity might not be a constant but a sensitively position-dependent quantity due to the occurrence of spin imbalance near the edges of the sample, and in order to take the effects of spin imbalance properly into account, a microscopic calculation of both the quantum dissipationless spin current and the spin accumulation based on an equal footing is thus required. We stress that some usual concepts about the interplay between spin current and spin imbalance might not be suitable for describing the intrinsic spin Hall effect. After some modifications, the theory presented in this paper might also be applied to investigate the effects of spin imbalance in the intrinsic spin Hall effect in 2DEGs with Rashba spinorbit coupling. Finally, it should be pointed out that though in the last year many theoretical works have been devoted to the study of the intrinsic spin Hall effect, many controversial issues still exist concerning some fundamental aspects of this extraordinary effect. Among them, a big controversial issue is that what is the correct definition of spin current in materials with intrinsic spin-orbit coupling. ${ }^{9,16,19,21}$ As was argued in Ref. 9 and in Ref. 19, there are some difficulties with the conventional definition of spin current in spin-orbit-coupled systems, but it seemed that up to now there are still no unanimous views about this question. ${ }^{9,16-19,21,23}$ (In the present paper we have used the same definition of Ref. 8.) Because no unambiguous experimental detections have ever been done, on the present stage such controversial issues are difficult to be clarified unambiguously by pure theoretical arguments. But it could be anticipated that by combining future experimental results with more accurate theoretical investigations, these controversial issues should be able to be clarified unambiguously in the near future.

\section{ACKNOWLEDGMENTS}

S.Q.S. is supported by a grant from the Research Grant Council of Hong Kong. L.B.H. is supported by the National Science Foundation of China (Grant No.10474022).
${ }^{1}$ G. A. Prinz, Science 282, 1660 (1998); S. A. Wolf, D. D. Awschalom, R. A. Buhrman, J. M. Daughton, S. Von Molnar, M. L. Roukes, A. Y. Chtchelkanova, and D. M. Treger, Science 294, 1488 (2001).

${ }^{2}$ P. R. Hammar, B. R. Bennett, M. J. Yang, and M. Johnson, Phys. Rev. Lett. 83, 203 (1999).

${ }^{3}$ G. Schmidt, D. Ferrand, L. W. Molenkamp, A. T. Filip, and B. J. Van Wees, Phys. Rev. B 62, R4790 (2000).

${ }^{4}$ Y. Ohno, D. K. Young, B. Beschoten, F. Matsukura, H. Ohno, and D. D. Awschalom, Nature (London) 402, 790 (1999).

${ }^{5}$ R. Fiederling, M. Keim, G. Reuscher, W. Ossau, G. Schmidt, A. Waag, L. W. Molenkamp, Nature (London) 402, 787 (1999).

${ }^{6}$ R. Mattana, J.-M. George, H. Jaffrès, F. Nguyen Van Dau, A. Fert, B. Lépine, A. Guivarc'h, and G. Jézéquel, Phys. Rev. Lett. 90, 166601 (2003).

${ }^{7}$ J. M. Luttinger, Phys. Rev. 102, 1030 (1956).

${ }^{8}$ S. Murakami, N. Nagaosa, and S.-C. Zhang, Science 301, 1348 (2003). Some supporting online material for the article was given in cond-mat/0308167 (unpublished).

${ }^{9}$ S. Murakami, N. Nagaosa, and S.-C. Zhang, Phys. Rev. B 69, 235206 (2004).

${ }^{10}$ J. E. Hirsch, Phys. Rev. Lett. 83, 1834 (1999).

${ }^{11}$ S. Zhang, Phys. Rev. Lett. 85, 393 (2000).

${ }^{12}$ L. B. Hu, J. Gao, and S. Q. Shen, Phys. Rev. B 68, 115302 (2003); 68, 153303 (2003).

${ }^{13}$ D. J. Thouless, M. Kohmoto, M. P. Nightingale, and M. den Nijs, Phys. Rev. Lett. 49, 405 (1982).

${ }^{14}$ Y. Hatsugai, Phys. Rev. Lett. 71, 3697 (1993).

${ }^{15}$ G. Sundaram and Q. Niu, Phys. Rev. B 59, 14915 (1999).

${ }^{16}$ J. Sinova, D. Culcer, Q. Niu, N. A. Sinitsyn, T. Jungwirth, and A. H. MacDonald, Phys. Rev. Lett. 92, 126603 (2004).

${ }^{17}$ S.-Q. Shen, M. Ma, X. C. Xie, and F. C. Zhang, Phys. Rev. Lett.
92, 256603 (2004).

${ }^{18}$ J. Schliemann and D. Loss, Phys. Rev. B 69, 165315 (2004).

${ }^{19}$ E. I. Rashba, Phys. Rev. B 68, 241315(R) (2003).

${ }^{20}$ S. Murakami, Phys. Rev. B 69, 241202(R) (2004).

${ }^{21}$ D. Culcer, J. Sinova, N. A. Sinitsyn, T. Jungwirth, A. H. MacDonald, and Q. Niu, cond-mat/0309475 (unpublished).

${ }^{22}$ N. A. Sinitsyn, E. M. Hankiewicz, W. Teizer, and J. Sinova, condmat/0310315 (unpublished).

${ }^{23}$ J. P. Hu, B. A. Bernevig, and C. Wu, cond-mat/0310093 (unpublished).

${ }^{24}$ Y. Xiong and X. C. Xie, cond-mat/0403083 (unpublished).

${ }^{25} \mathrm{~J}$. Inoue, G. E. W. Bauer, and L. W. Molenkamp, cond-mat/ 0402442 (unpublished).

${ }^{26}$ O. V. Dimitrova, cond-mat/0405339 (unpublished).

${ }^{27}$ A. A. Burkov and A. H. MacDonald, cond-mat/0311328 (unpublished).

${ }^{28}$ W. P. Pratt, Jr., S. F. Lee, J. M. Slaughter, R. Loloee, P. A. Schroeder, and J. Bass, Phys. Rev. Lett. 66, 3060 (1991).

${ }^{29}$ M. A. M. Gijs, S. K. J. Lenczowski, and J. B. Giesbers, Phys. Rev. Lett. 70, 3343 (1993).

${ }^{30}$ M. Johnson and R. H. Silsbee, Phys. Rev. Lett. 55, 1790 (1985); M. Johnson, ibid. 70, 2142 (1993); M. Johson and R. H. Silsbee, Phys. Rev. B 35, 4959 (1987).

${ }^{31}$ T. Valet and A. Fert, Phys. Rev. B 48, 7099 (1993).

${ }^{32}$ C. Heide, Phys. Rev. B 65, 054401 (2001).

${ }^{33}$ Semiconductor Spintronics and Quantum Computation, edited by D. D. Awschalom, D. Loss, and N. Samarth (Springer, New York, 2002).

${ }^{34}$ Y. Kato, R. C. Myers, A. C. Gossard, and D. D. Awshalom, condmat/0403407 (unpublished).

${ }^{35}$ J. Stephens, J. Berezovsky, J. P. McGuire, L. J. Sham, A. C. Gossard, and D. D. Awschalom, cond-mat/0401197 (unpublished). 
${ }^{36}$ J. Inoue, G. E. W. Bauer, and L. W. Molenkamp, Phys. Rev. B 67, 033104 (2003).

${ }^{37}$ V. M. Edelstein, Solid State Commun. 73, 233 (1990).

${ }^{38}$ When an in-plane electric field is applied in a two-dimensional electron gas with Rashba spin-orbit coupling, the spin-orbit coupling itself (without resort to the boundary effects) will induce a homogeneous spin accumulation with the spin-polarization direction perpendicular to the electric field. This effect was called the kinetic magnetoelectric effect (Refs. 36 and 37). This effect should not be confused with the usual spin accumulation phenomena widely studied in the literatures. Form theoretical viewpoints, the usual spin accumulation phenomena are caused by the boundary effects (Refs. 28-33), but the kinetic magnetoelectric effect is caused by the combined action of the spin-orbit coupling and the electric field and has no relation with the boundary effects. 